\title{
Violencia contra las mujeres y libertad de expresión: tensiones jurídicas
}

\section{Larissa Arroyo Navarrete ${ }^{1}$}

\section{Resumen}

Recepción: 6 de julio de 2017 / Aprobación: 29 de setiembre de 2017

La objetivización y la sexualización de las mujeres en los medios de comunicación y la publicidad deriva en la naturalización de la violencia hacia estas a través del humor y otras manifestaciones culturales. Si bien existe un marco internacional de derechos humanos que provee de protección especial a las mujeres, este se contrapone con la protección jurídica que también recibe la libertad de expresión, como pilar de los sistemas democráticos. Es urgente que en Costa Rica se haga una reforma legislativa que reconozca los deberes del Estado de cara al derecho a la igualdad y a la no discriminación, así como también los límites de la libertad de expresión cuando, a través del uso de roles y estereotipos de género y de la sexualización de los cuerpos de las mujeres en los medios de comunicación y la publicidad, esta libertad impacta de manera estructural y sistemática como violencia por la condición de género, tal y como ocurre en el caso emblemático del programa radial Manicomio de la Risa.

\section{Palabras clave}

Derechos humanos; género; violencia sexual; medios de comunicación; igualdad

\section{Abstract}

Objectification and sexualization of women in the media and advertising involves the naturalization of violence towards them through humor and other traditions. While there is an international human rights framework which provides special protection for women, this contrasts with the legal protection freedom of expression also receives as a pillar of our democratic systems. In Costa Rica, it is urgent to have legislation that identifies the duties of the Costa Rican government regarding the right to equality and non-discrimination and to live free of violence, as well as the limits of freedom of expression when, through the use of roles and gender stereotypes and the sexualization of women's bodies in the media and advertising, this freedom has a historical, structural and systematic impact as gender based violence, like in the emblematic case of the radio program Manicomio de la Risa.

\section{Keywords}

Human rights; gender; sexual violence, media, equality

1 Costarricense. Máster en Derechos Humanos por la Universidad Estatal a Distancia de Costa Rica (UNED). Abogada especialista en Derechos Humanos y Género, Asociación Ciudadana ACCEDER (Acciones Estratégicas por los Derechos Humanos). Correo electrónico: larissa.arroyo@gmail.com 


\section{Resumo}

A objetivização e a sexualização das mulheres na publicidade e nos meios de comunicação acarretam a naturalização da violência a elas, através do humor e outras tradições. Embora exista um marco internacional de direitos humanos, que provê uma proteção especial para as mulheres, este se contrapõe à proteção jurídica, que também recebe a liberdade de expressão como pilar dos sistemas democráticos. É urgente que se faça na Costa Rica uma reforma legislativa que reconheça os deveres do Estado costa-riquenho face ao direito à igualdade e à não discriminação. Essa reforma deve também repensar os limites da liberdade de expressão, quando esta através do uso de padrões e estereótipos de gênero e da sexualização dos corpos das mulheres nos meios de comunicação e na publicidade impacta como violência pela condição de gênero, de modo estrutural e sistemático. Tal como acontece no caso emblemático do programa de rádio Manicomio de la Risa

\section{Palavras chave}

Direitos humanos; gênero; violência sexual; mídias de comunicação; igualdade

\section{Introducción}

"Cachetón, ¿qué buena acción hiciste hoy?"

"iEvité que violaran a una muchacha!"

"¿En serio? ¿Y cómo? ¿Qué pasó?"

"iAh, es que la logré convencer!"

(Manicomio de la Risa, 23 de febrero de 2015).

Existe todo un abanico de diferencias entre la cotidianidad en la que viven los hombres y aquella que viven las mujeres. Para efectos del presente análisis, tal situación se resume en la expresión "riesgo constante". Esta alude a la amenaza permanente que enfrentan las mujeres, por ejemplo, ante la posibilidad de ser víctimas de acoso sexual en los espacios públicos y privados, de hostigamiento sexual -en los ámbitos laboral y educativo- y de ser víctima, particularmente, durante la niñez y la adolescencia, de abusos e incluso violación sexual por un conocido o familiar; no obstante, destaca la amenaza de ser responsabilizadas del delito del que fueron víctimas, o bien, de vivir entre costumbres y tradiciones disfrazadas de humor y folklorismo para naturalizar una explícita apología de la violencia contra las mujeres.

Las mujeres viven historias trágicas que se convierten en bromas en los medios de comunicación y la publicidad, las cuales sacan sonrisas y risotadas y, algunas otras veces, sacan también el dinero, porque dichas historias de violencia son "el gancho" con que lucra gran parte del sector comercial. El humor, el folklor, e incluso el glamour, son instrumentos claves de los medios de comunicación y de la publicidad para atrincherarse desde la legitimidad socio-cultural e implantar o sostener la idea de que las mujeres debemos 
estar sujetas a la voluntad y deseo de alguien más, que nuestros cuerpos no son nuestros para disponer de ellos, que las decisiones sobre nuestra sexualidad y capacidad reproductiva no son nuestras y que el perdón de nuestras vidas - para no morir a manos de nuestro compañero sentimental, o del que desea serlo- depende de nuestro grado de anuencia a no actuar como sujetas libres.

Este artículo pretende ser un estudio crítico y tiene como objetivo principal determinar y analizar el marco jurídico de protección especial a las mujeres, en el Derecho Internacional de los Derechos Humanos, y los consecuentes deberes del Estado costarricense del derecho a la igualdad y a la no discriminación, así como de vivir libre de violencia y, en relación con la libertad de expresión y los límites de esta, identificar las violaciones de estos derechos -a través del uso de roles y estereotipos de género, así como la sexualización de los cuerpos de las mujeres en los medios de comunicación y la publicidad- y determinar las deficiencias y necesidades de reformas de la normativa nacional, para eliminar el fenómeno histórico, estructural y sistemático de la violencia contra las mujeres, tomando como caso emblemático el programa radial Manicomio de la Risa.

Se parte de la hipótesis de que, aunque el respeto a la libertad de expresión es fundamental para asegurar las democracias, sin que por ello esta deba de ser entendida como irrestricta o ilimitada, el ejercicio de dicha libertad individual puede llegar a violentar derechos fundamentales y humanos como, por ejemplo, el de vivir una vida libre de violencia, la igualdad y la no discriminación. Por lo tanto, es urgente impulsar políticas públicas que apunten a erradicar los contenidos sexistas, estereotipados y discriminatorios, para desarrollar, desde una perspectiva de derechos humanos y de género, la tensión que existe actualmente entre los derechos de las mujeres reconocidos en instrumentos internacionales -tales como el derecho a vivir libre de violencia y el derecho a la igualdad y no discriminación-, en contraposición con la libertad de expresión como derecho humano, cuyos límites pueden ser dados precisamente por la protección jurídica especial a las mujeres.

Para analizar dichas dinámicas, se recurrirá a un ejercicio de ponderación, considerando la normativa internacional de derechos humanos y, en particular, aquella que establece el deber estatal de reconocer y brindar una protección especial a las mujeres y, desde el principio y el derecho de igualdad y no discriminación amparado constitucionalmente, como categoría prohibida de discriminación, así como el derecho a vivir libre de violencia, en contraposición a la protección jurídica de la libertad de expresión. Además, se incorporará el criterio de los órganos encargados de realizar la interpretación legítima de estos cuerpos normativos a través de observaciones y recomendaciones y, principalmente, de la jurisprudencia de la Corte Interamericana de Derechos Humanos (CIDH). 
Por último, con base en este marco normativo y en las conclusiones resultantes sobre los deberes estatales sobre la materia, se hará referencia a un caso paradigmático en materia de violencia contra las mujeres, el cual permite ejemplificar la objetivización y sexualización² de la imagen de estas; me refiero al programa radial costarricense El Manicomio de la Risa ${ }^{3}$, de Omega Estéreo, emisora del Grupo Radiofónico Omega. El análisis de este programa ha permitido demostrar las deficiencias de nuestro sistema normativo y su implementación, dada la existencia de violencia mediática y, en particular, del fenómeno de banalización de la violencia sexual. Se hará hincapié en la responsabilidad jurídica tanto del Estado costarricense por fallar en brindar una efectiva protección jurídica, como la de los medios de comunicación, pues contribuyen a la perpetuación de roles y estereotipos de género -los cuales sustentan la violencia contra las mujeres en todas sus manifestaciones-, como también la responsabilidad de los medios de comunicación, por ser una práctica que, desde la cotidianidad, perpetúa la dominación, la desigualdad y la discriminación en las relaciones sociales que naturalizan la subordinación de las mujeres en la sociedad y vulneran tanto el derecho humano de todas las mujeres a vivir libres de violencia, así como el derecho a la igualdad y a la no discriminación.

\section{Marco jurídico internacional de protección especial a las mujeres}

La Sala Constitucional costarricense ha mantenido una línea jurisprudencial de reconocimiento del carácter supraconstitucional de los instrumentos internacionales de derechos humanos, en la medida en que estos brinden mayor cobertura o tutela de un determinado derecho. Esta es una construcción efectuada por esta entidad constitucional a partir del Artículo $7^{4}$ y de los

2 La objetivación y la sexualización es una práctica socio-cultural discriminatoria que se sostiene a partir de roles y estereotipos de género que perpetúan la subordinación y desigualdad de las mujeres en la sociedad. Los Estados tienen la obligación jurídica internacional y nacional de reconocer las discriminaciones y las violencias que viven las mujeres y tomar acciones para modificar estas prácticas, incluyendo la modificación de su normativa, en tanto que las mujeres sean reducidas a una condición de cuerpos/objetos sexualizados, por ejemplo, en el uso de su imagen en la publicidad como parte captatoria, deniega su condición de personas y como tales son titulares de derechos.

3 El Manicomio de la Risa es un programa que se presenta como humorístico, el cual se transmite por Omega Estéreo, de lunes a viernes, de 6:00 a. m. a 9:00 a. m., conducido por Ronny "La Perla" Zelaya, en el dial 105.1 FM. Dicho programa está dirigido a una audiencia amplia y popular. El mismo hace uso del humor, o lo que se presenta como tal, para hacer mofa a partir de contenidos misóginos y sexistas, así como homolesbobitransfobicos, entre otros.

4 "ARTíCULO 7․- Los tratados públicos, los convenios internacionales y los concordatos, debidamente aprobados por la Asamblea Legislativa, tendrán desde su promulgación o desde el día que ellos designen, autoridad superior a las leyes". Los tratados públicos y los convenios internacionales referentes a la integridad territorial o la organización política del país, requerirán de la aprobación de la Asamblea Legislativa, por votación no menor de las tres cuartas partes de la totalidad de sus miembros, y la de los dos tercios de los miembros de una Asamblea Constituyente, convocada al efecto" (Constitución Política de la República de Costa Rica). 
alcances del Artículo $48^{5}$ de la Constitución Política de la República de Costa Rica (Orozco, 2014, p. 11). A modo de ejemplo, el voto constitucional 13.254, del año 2015, establece que:

Asimismo, a favor de los menores de edad, el derecho a la educación es reconocido en diversos instrumentos internacionales vigentes en nuestro país, con valor incluso superior a las leyes que les reconocen los Artículos 7 y 48 de la Constitución Política (Orozco, 2014, p. 15).

En ese sentido, es imprescindible tomar nota sin que esta constituya una lista taxativa de algunos de los instrumentos fundamentales que reconocen y brindan cobertura a los derechos humanos de las mujeres y, en particular, aquellos que constituyen una protección especial a esta población históricamente vulnerada, tales como la Convención Americana de Derechos Humanos (CADH), Convención sobre la Eliminación de Todas las Formas de Discriminación contra la Mujer (CEDAW) y Convención Interamericana para Prevenir, Sancionar y Erradicar la Violencia contra la Mujer (Convención Belém do Pará). En otras palabras, si bien nuestra normativa nacional puede resultar deficiente en cuanto a la protección especial a las mujeres en la temática que trata el presente estudio, resulta imprescindible considerar que estos otros instrumentos internacionales son parte de nuestro bloque jurídico, al punto de establecer incluso el deber jurídico de legislar para hacerlos efectivos. Si bien es cierto que existen múltiples derechos que son violentados por la falta de legislación específica para sancionar el uso de roles y estereotipos de género -así como de la imagen de la mujer para objetivizarla o sexualizarla en los medios de comunicación y la publicidad- haremos énfasis en la violación al derecho a la igualdad y a la no discriminación, así como al derecho de las mujeres a vivir libre de violencia.

Además, se ha de mencionar que un mecanismo que, actualmente, tutela la protección de la imagen de las mujeres es la Ley 5.811 de 1975, la cual regula la propaganda que utilice la imagen de la mujer con el correspondiente Reglamento a esta ley, bajo mandato de hacerla efectiva por la Oficina de Control de Propaganda, como dependencia del Ministerio de Gobernación y Policía. Sin embargo, esta normativa se restringe como norma, tal y como establece el artículo primero de esta legislación, a todo tipo de propaganda comercial que ofenda la dignidad, el pudor de la familia y en la que se utilice la imagen de la mujer de manera inmodesta, para promover las ventas.

5 "ARTÍCULO 48.- Toda persona tiene derecho al recurso de hábeas corpus para garantizar su libertad e integridad personales, y al recurso de amparo para mantener o restablecer el goce de los otros derechos consagrados en esta Constitución, así como de los de carácter fundamental establecidos en los instrumentos internacionales sobre derechos humanos, aplicables a la República. Ambos recursos serán de competencia de la Sala indicada en el artículo 10" (Constitución Política de la República de Costa Rica). 


\section{Derechos en conflicto}

\section{Derecho a la igualdad \\ y ¿a la no discriminación?}

El principal instrumento que recoge normativa para la protección especial de las mujeres y, en particular, su derecho a la igualdad y a la no discriminación, es la Convención CEDAW. En el artículo primero de este cuerpo normativo se establece que:

la expresión "discriminación contra la mujer" denotará toda distinción, exclusión o restricción basada en el sexo que tenga por objeto o por resultado menoscabar o anular el reconocimiento, goce o ejercicio por la mujer, independientemente de su estado civil, sobre la base de la igualdad del hombre y la mujer, de los derechos humanos y las libertades fundamentales en las esferas política, económica, social, cultural y civil o en cualquier otra esfera (CEDAW).

Interesa el artículo segundo que establece el deber de los Estados de abstenerse de incurrir en todo acto o práctica de discriminación contra la mujer y velar porque las autoridades e instituciones públicas actúen de conformidad con esta obligación. Pero, sobre todo, insta a tomar todas las medidas apropiadas para eliminar la discriminación contra la mujer practicada por cualesquiera personas, organizaciones o empresas, y adaptar todas las medidas adecuadas, incluso de carácter legislativo, para modificar o derogar leyes, reglamentos, usos y prácticas que constituyan discriminación contra la mujer. Tampoco puede ser obviado el Artículo 5, referente al deber estatal de

modificar los patrones socioculturales de conducta de hombres y mujeres, con miras a alcanzar la eliminación de los prejuicios y las prácticas consuetudinarias y de cualquier otra índole que estén basados en la idea de la inferioridad o superioridad de cualquiera de los sexos o en funciones estereotipadas de hombres y mujeres (CEDAW).

Incluso, la recomendación general No. 19 del Comité de la CEDAW sobre Violencia contra la mujer de 1992, establecía, en su inciso d), que se adoptaran medidas eficaces para garantizar que los medios de comunicación respeten a la mujer y promuevan el respeto hacia esta, como en su inciso i), el cual establece los procedimientos eficaces de denuncia y reparación, incluida la indemnización.

\section{Derecho a vivir libre de violencia}

Partiremos de la definición de violencia que da la Convención Interamericana para Prevenir, Sancionar y Erradicar la Violencia contra la Mujer (Convención de Belém do Pará), la cual determina en su artículo primero que:

para los efectos de esta Convención debe entenderse por violencia contra la mujer cualquier acción o conducta, basada en su género, que cause 
muerte, daño o sufrimiento físico, sexual o psicológico a la mujer, tanto en el ámbito público como en el privado.

Lo anterior es complementado por el artículo siguiente, el cual establece que la violencia contra la mujer incluye la violencia física, sexual y psicológica que sea perpetrada por cualquier persona y que sea cometida o tolerada por el Estado o sus agentes, dondequiera que esta ocurra. De esta normativa se deriva, precisamente, la obligación del Estado de asegurar los derechos que todas las mujeres, incluyendo el derecho a una vida libre de violencia, tanto en el ámbito público como en el privado (Artículo 3), así como el derecho a ser libre de toda forma de discriminación, y a ser valorada y educada libre de patrones estereotipados de comportamiento y prácticas sociales y culturales basadas en conceptos de inferioridad o subordinación (Artículo 6).

De manera explícita, el Artículo 7 establece el deber de los Estados, incluyendo a Costa Rica como signataria, de 1) adoptar, por todos los medios apropiados y sin dilaciones, políticas orientadas a prevenir, sancionar y erradicar dicha violencia; 2) abstenerse de cualquier acción o práctica de violencia contra la mujer; 3) actuar con la debida diligencia para prevenir, investigar y sancionar la violencia contra la mujer; 4) incluir en su legislación interna normas penales, civiles y administrativas, así como las de otra naturaleza, que sean necesarias para prevenir, sancionar y erradicar la violencia contra la mujer; 5) adoptar las medidas administrativas que sean concernientes al caso, incluyendo las de tipo legislativo, para modificar o abolir leyes y reglamentos vigentes, o para modificar prácticas jurídicas o consuetudinarias que respalden la persistencia o la tolerancia de la violencia contra la mujer; 6) establecer procedimientos legales justos y eficaces para la mujer que haya sido sometida a violencia; 7) establecer los mecanismos judiciales y administrativos necesarios para asegurar que la mujer objeto de violencia tenga acceso efectivo a resarcimiento, reparación del daño u otros medios de compensación justos y eficaces, y 8) adoptar las disposiciones legislativas o de otra índole que sean necesarias para hacer efectiva esta Convención (Artículo 7).

No obstante, la violencia contra las mujeres es un hecho cotidiano invisibilizado y normalizado y las mujeres seguimos siendo objetivizadas y puestas al antojo de un otro, sin poder decidir sobre nuestros cuerpos y sin ser reconocidas como sujetas plenas de derechos, al punto de que "hemos naturalizado esta violencia y su causa estructural es innombrable: el patriarcado que comemos cada día en nuestras familias, en nuestros trabajos, en nuestros centros de educación, en nuestras calles" (Arroyo Navarrete, 2015a, párr. 2).

La cotidianidad de un "humor" como el del Manicomio de la Risa constituye un tipo de violencia mediática ya que, al sostener la reproducción de roles, patrones y estereotipos de género, perpetúa la dominación, la desigualdad y la discriminación en las relaciones sociales, naturaliza la subordinación de 
las mujeres en la sociedad y vulnera el derecho humano de todas las niñas, adolescentes, adultas y adultas mayores, a vivir libres de violencia (Arroyo Navarrete, 2015b).

A la luz de los artículos citados de la Convención Belém Do Pará, es flagrante la violación al derecho a vivir libre de violencia de las mujeres en Costa Rica. Ello resulta del fallo por parte del Estado costarricense en legislar para establecer una normativa que permita a las mujeres contar los mecanismos idóneos y efectivos para el acceso a la justicia en casos como estos, en los cuales se emplea la imagen de la mujer para su sexualización, objetivización y explotación, así como de la perpetuación de estereotipos y roles de género que legitiman o exacerban la violencia contra las mujeres y las prácticas que se basen en la premisa de la inferioridad o superioridad de cualquiera de los géneros.

También, se ha de considerar la omisión del cumplimiento de Costa Rica del Artículo 8, inciso g, el cual establece el deber de los Estados de "alentar a los medios de comunicación a elaborar directrices adecuadas de difusión que contribuyan a erradicar la violencia contra la mujer en todas sus formas y a realzar el respeto a la dignidad de la mujer" (Convención Belém do Pará, 1979, Artículo 8).

\section{Libertad de expresión}

Si bien es cierto, el respeto a la libertad de expresión es fundamental en democracias como la costarricense, al igual que el resto de los otros derechos humanos y libertades fundamentales, esta no puede ser irrestricta o ilimitada porque la libertad de expresión es un ejercicio de la libertad individual. Este puede tener límites que se justifican en la protección del orden público y la seguridad nacional y también en derechos como el honor, la intimidad y el derecho a vivir libre de violencia (Arroyo Navarrete, 2015).

En los casos, por ejemplo, de injurias, calumnias o difamación, no se suele cuestionar la razón, por la cual es inadecuado decir algo sobre otra persona que sea falso o degradante. Sin embargo, la sociedad ha naturalizado el imaginario de violencia y, en particular, contra las mujeres, pero también contra otras poblaciones históricamente vulneradas, como personas migrantes o extranjeras, personas lesbianas, bisexuales, gais, trans, personas afro, indígenas, personas con discapacidad, entre otras. Recordar que existen límites a la libertad dados -incluso por la misma normativa internacional de derechos humanos- es imprescindible para la propuesta de reforma legislativa nacional. De ahí la importancia de mencionar que tanto la Comisión como la Corte Interamericana de Derechos Humanos han señalado:

que el derecho a la libertad de expresión no es absoluto y puede estar sujeto a ciertas limitaciones, según los incisos 2, 4 y 5 del artículo 13 de la Convención Americana, las cuales, para ser legítimas, deben cumplir una serie de condiciones específicas. Particularmente, el artículo 13.2 exige el cumplimiento de tres condiciones básicas para que una limitación al derecho a la libertad de expresión sea admisible: 1) la limitación debe haber sido definida en forma precisa y clara 
a través de una ley formal y material, 2) la limitación debe estar orientada al logro de objetivos legítimos autorizados por la Convención Americana, y 3) la limitación debe ser necesaria en una sociedad democrática para el logro de los fines legítimos que se buscan; estrictamente proporcional a la finalidad perseguida; e idónea para lograr el objetivo imperioso que pretende lograr (CIDH, 2013, párr. 68).

Un punto esencial, en ese sentido, radica en que estas mismas entidades interamericanas de derechos humanos han establecido que:

para ser admisibles, las limitaciones deben estar establecidas mediante responsabilidades ulteriores por el ejercicio del derecho, resultando inadmisibles las limitaciones previas (censura), aquellas que produzcan efectos discriminatorios y que se impongan a través de mecanismos indirectos como los que proscribe el artículo 13.3 de la Convención Americana (CIDH, 2013).

Queda claro que la propuesta de reforma legislativa no puede derivarse de un contenido que sancione anteriormente al cometido de los hechos, es decir, que no puede promoverse la censura previa pero sí mecanismos de sanción posterior.

\section{Resultado de la ponderación}

Hay que recordar que los responsables principales del cumplimiento de los derechos humanos son los Estados y que es la comunidad internacional la garante de estos cuando las diferentes entidades incumplen sus deberes (Beitz, 2012, p. 13). Es decir, son los principales responsables, pero, dentro de esas obligaciones, se encuentra -como se ha descrito en los textos normativos internacionales de derechos humanos- el deber de tutelar y de velar íntegramente por el cumplimiento de tales derechos, con lo cual deben de asumir acción ante la conducta de terceros que puedan vulnerarlos.

Sin duda alguna, nos encontramos ante un ejercicio en el que hay que considerar el poder de los medios de comunicación y que, por lo tanto, ha impedido la discusión integral del tratamiento discriminatorio siempre bajo el argumento de la necesidad vital del derecho a la libertad de expresión. Incluso, en la misma normativa internacional, se ha visto con timidez este tema. Por ejemplo, en la Conferencia de la Mujer, celebrada en México en 1975, se discutió su papel fundamental en la transmisión de valores y en la discriminación en los roles de género, y la subsecuente necesidad de instar a las empresas a mejorar la imagen de esta población para poder sumarlas en los cargos de decisión. Posteriormente, en Copenhague, en el año 1980, y en Nairobi, en 1985, se abordó el tema, pero no se explicitó, en ninguno, la presente propuesta de la urgencia de una normativa para su regulación. Ya para la IV Conferencia Mundial de la Mujer realizada por las Naciones Unidas, en Beijing en 1995, dentro de las 12 áreas de interés prioritario para lograr el objetivo de la igualdad de género se consideraron los medios de comunicación. Al respecto, se establece que: 
la libertad de expresión, por un lado, es un instrumento idóneo para que todas las personas -por ser individuos igualmente 'dignos'- puedan manifestar sus ideas, emociones, sentimientos, intereses, etc. Desde esta perspectiva, la libertad de expresión supone el reconocimiento de la igual dignidad de los seres humanos y, simultáneamente, se ofrece como un medio para que dicho reconocimiento se haga patente. El derecho a no ser discriminado, por su parte, representa una garantía de que todas las personas -precisamente por tener la misma 'dignidad'- recibirán un trato igual en circunstancias similares. En este sentido, la prohibición de toda discriminación injustificada es reflejo del reconocimiento de la dignidad personal y, al mismo tiempo, constituye una garantía para la afirmación efectiva de la misma (Salazar y Gutiérrez, 2008, p. IX).

Hay una tendencia marcada a glamurizar la libertad de expresión. Si bien comparto el criterio de la Comisión Interamericana de Derechos Humanos y la Corte Interamericana de Derechos Humanos acerca de la imposibilidad de la censura previa en un contexto democrático, hemos de recordar que la violencia contra las mujeres es una pandemia que genera deberes para los Estados por sus acciones y por las de terceros. Esto significa que, en un ejercicio de ponderación, es inevitable, por un lado, responder a los estándares jurídicos internacionales en materia de libertad de expresión; no obstante, por el otro, resulta necesario establecer, de manera clara, y por ley, los límites y la descripción precisa de las justificaciones. No obstante, si lo que se pretende salvaguardar con el principio de la libertad de expresión es el contexto democrático, la libertad y la dignidad de las personas -y tomando en cuenta el peso histórico de la violencia contra las mujeres y el no reconocimiento de ellas como sujetas plenas de derecho, incluyendo las debidas protecciones-, no podemos obviar que, hasta el momento, no solo no se han tomado acciones para cumplir con los deberes estatales, sino que ni siquiera hemos dado la discusión sobre el claro desbalance existente en materia de los derechos humanos, donde algunas son consideradas más personas que otras.

Si bien existe una diferencia entre los medios de comunicación, es de gran relevancia citar el ejemplo del análisis que hizo la CIDH en un caso de discriminación contra las mujeres en los medios de comunicación de la sentencia de la Sala Primera de la Corte Suprema de Justicia de Costa Rica, número 198, expediente 06-000100-0163-CA, del 4 de febrero de 2010. La misma versa sobre la competencia del Instituto sobre Alcoholismo y Farmacodependencia (IAFA) para restringir el uso de la imagen de la mujer en el material publicitario de una bebida alcohólica, como es la cerveza Pilsen. En este caso, la Sala falló a favor del IAFA con base en consideraciones relacionadas con los derechos de las mujeres a vivir libres de discriminación y la protección de su integridad y dignidad, refiriéndose a la Convención Americana y la CEDAW, entre otros instrumentos internacionales. La opinión que le mereció a la $\mathrm{CIDH}$ es de gran valor para la discusión, ya que estableció que:

Consecuentemente, si el Estado costarricense posee el compromiso y la obligación de evitar aquellas prácticas que atenten contra la imagen de la mujer, 
y siendo que lo presentado ante el IAFA fueron unas fotografías donde se representa a la mujer como instrumento u objeto, pues el perfil femenino utilizado explota las características físicas, anatómicas de las modelos, el Instituto está en la obligación de prohibir el material. Ciertamente, puede pensarse que el respeto a la imagen de la mujer es un tema metajurídico, empero aún con esa posición, lo cierto es que ello impregna el derecho, pues representa los valores de una sociedad en un momento determinado y como tal han de ser respetados por la colectividad. Atendiendo a lo expuesto, la mayoría de esta Sala considera que la propaganda citada atenta contra la imagen de la mujer, por ende, el IAFA actuó correctamente al denegarla (CIDH, 2011, p. 112).

Si bien este fallo tiene un fondo de cumplimiento de la normativa internacional de derechos humanos, no se puede negar la actual urgencia en la atención al flagelo de la violencia contra las mujeres, ya que esta sentencia es la excepción y no la norma en el país.

\section{Conclusiones}

El caso del Manicomio de la Risa es paradigmático, ya que precisamente apunta al fenómeno de la violencia contra las mujeres y sus mecanismos de perpetuación como algo estructural, sostenido en el tiempo y cotidiano. Es obligación de los Estados, incluyendo el costarricense, el no lesionar la libertad de expresión, pero también lo es velar por los derechos humanos de las mujeres. En esta pugna jurídica es imperativo considerar que nuestra normativa incluye los mandatos y deberes de los instrumentos internacionales de derechos humanos, para entender la urgencia de la adopción de políticas públicas que apunten a erradicar los contenidos sexistas, estereotipados, discriminatorios, homo-lesbo-bi-transfóbicos, xenofóbicos y racistas en los medios de comunicación y la publicidad.

Si bien, la normativa nacional es insuficiente para prevenir y erradicar la violencia contra las mujeres -y en particular aquella que es ejercida por los medios de comunicación-, en Costa Rica se cuenta con el derecho internacional de derechos humanos y ciertos instrumentos de protección especial a las mujeres que dejan clara la necesidad de reforma legislativa nacional para la prevención y sanción del uso de la imagen de las mujeres para la sexualización y objetivización de estas, así como la perpetuación de roles y estereotipos de género que sostienen un sistema patriarcal bajo la diseminación de conceptos de inferioridad o subordinación basados en el género. En ese sentido, existe una violación a diversos derechos humanos de las mujeres, pero, en particular, a los derechos a vivir libre de violencia de cualquier tipo y al derecho a la igualdad y a la no no discriminación a través del trato diferenciado precisamente reflejado en esos roles, patrones y estereotipos de género. 
Cuando los medios de comunicación hacen caso omiso a su responsabilidad para transformar la cultura y la sociedad, incurren también en responsabilidad jurídica a pesar de que no exista normativa nacional, pero de manera particular, esta responsabilidad también recae en los Estados como el costarricense por no tomar las medidas necesarias para evitar que esto ocurra, incluyendo la debida diligencia y la creación de mecanismo de acceso a la justicia para las mujeres.

El caso emblemático del programa radial Manicomio de la Risa deja claro que, en estos momentos, toleramos la violencia contra las mujeres por percibirla como algo natural y cotidiano que, por lo demás, se ve adornada de supuesto humor y folklor sin prever las nefastas consecuencias e impacto que eso tiene en la vida de las mujeres. Resulta imperativo, por lo tanto, la discusión de un proyecto de ley que norme la conducta de los medios de comunicación para sancionar, no de manera previa, sino posterior, las conductas discriminatorias y violatorias de los derechos humanos de las mujeres, así como promover políticas y directrices adecuadas de difusión que contribuyan a erradicar la violencia contra la mujer en todas sus formas y a respetar de manera integral sus derechos humanos y libertades fundamentales.

\section{Referencias}

Arroyo Navarrete, Larissa. (2015a). El machismo mata, el feminismo no. Revista Paquidermo. Recuperado de http://www.revistapaquidermo.com/ archives/12757

Arroyo Navarrete, Larissa. (2015b). Violencia contra mujeres: un límite a la libertad de expresión. Diario Extra. Recuperado de http://www.diarioextra.com/Noticia/detalle/256579/violencia-contra-mujeres:--un-limite-ala-libertad-de-expresion

Asamblea Legislativa de la República de Costa Rica. (s. f.). Ley 5811 de Control de Propaganda que Utilice la Imagen de la Mujer. Recuperado de http://www.colper.or.cr/userfiles/file/juridico/publicidad/ley_5811.doc

Asamblea Legislativa de la República de Costa Rica. (s. f.). Reglamento Ley de Control a Propaganda con Imagen de la Mujer. Recuperado de http://www.colper.or.cr/userfiles/file/juridico/publicidad/11235-G.doc

Beitz, Charles. (2012). La idea de los derechos humanos. Madrid: Marcial Pons.

Comisión Interamericana de Derechos Humanos [CIDH]. (2011). Estándares jurídicos vinculados a la igualdad de género y a los derechos de las mujeres en el sistema interamericano de derechos humanos: desarrollo y aplicación. Recuperado de https://www.oas.org/es/cidh/mujeres/docs/pdf/ 
Comisión Interamericana de Derechos Humanos. (2013). Relatoría Especial para la Libertad de Expresión. Jurisprudencia nacional sobre libertad de expresión y acceso a la información. Recuperado de http://www.oas.org/ es/cidh/expresion/docs/publicaciones/2013\%2005\%2020\%20JURISPRUDENCIA\%20DOMESTICA\%20SOBRE\%2OLIBERTAD\%20DE\%20EXPRESION\%20Y\%20AI.pdf

Convención Interamericana para Prevenir, Sancionar y Erradicar la Violencia contra la Mujer (Convención de Belém do Pará). (1994). Recuperado de http://www.oas.org/es/mesecvi/docs/BelemDoPara-ESPANOL.pdf

Convención sobre la Eliminación de Todas las Formas de Discriminación contra la Mujer [CEDAW]. (1979). Recuperado de http://www.un.org/ womenwatch/daw/cedaw/text/sconvention.htm

Departamento de Derecho Internacional [DEA]. Convención Americana de Derechos Humanos. (s. f.). Recuperado de http://www.oas.org/dil/esp/ tratados_B-32_Convencion_Americana_sobre_Derechos_Humanos.htm

Orozco Solano, Víctor. (setiembre de 2014). El valor normativo de los Instrumentos Internacionales en materia de Derechos Humanos en el Sistema de Justicia Constitucional Costarricense: El caso particular de la jurisprudencia de la Corte Interamericana de Derechos Humanos. En Revista Judicial, 113. Recuperado de http://sitios.poder-judicial.go.cr/escuelajudicial/documentos/revs_juds/Revista\%20113/PDFs/02_archivo.pdf

Sala Constitucional de Costa Rica. (s. f.). Voto constitucional 13254 del 25 de agosto del 2015. 15-009521-0007-CO. Recuperado de http:// jurisprudencia.poder-judicial.go.cr/SClJ_PJ/busqueda/jurisprudencia/ jur_Documento.aspx?param1=Ficha_Sentencia\&nValor1=1\&nValor2=64 6868\&strTipM=T\&strDirSel=directo

Salazar Ugarte, Pedro y Gutiérrez Rivas, Rodrigo. (2008). El derecho a la libertad de expresión frente al derecho a la no discriminación. Instituto de Investigaciones Jurídicas de la Universidad Nacional Autónoma de México; Consejo Nacional para Prevenir la Discriminación. México D. F. Recuperado de http://biblio.juridicas.unam.mx/libros/libro.htm?l=2583 